\title{
Concepções de gestantes sobre o pré-natal realizado por profissional do Programa Mais Médicos
}

\author{
Pregnant women conception about prenatal care \\ performed by professional of "More Doctors" program
}

\begin{abstract}
Carine Amabile Guimarães, ${ }^{1}$ Narciso Vieira Soares, ${ }^{1}$ Juliane Pereira Dorneles, ${ }^{1}$ Eduarda Batista Kreuning 'Universidade Regional Integrada do Alto Uruguai e das Missões - Campus de Santo Ângelo, RS, Brasil.
\end{abstract}

Recebido em: 21/08/2016 / Aceito em: 15/09/2016 / Publicado em: 18/10/2016

dudabatistak@hotmail.com

\section{RESUMO}

Objetivo: identificar as concepções de gestantes sobre o pré-natal, realizado pelos médicos do Programa Mais Médicos, em uma UBS. Método: trata-se de uma pesquisa de caráter exploratório com uma abordagem qualitativa. Participaram do estudo 14 gestantes atendidas pelo SUS. Para a coleta de dados, utilizou-se a entrevista individual com um questionário norteador com questões abertas e fechadas, sendo realizadas na sala de consulta de enfermagem da Unidade Básica de Saúde, no período de junho e julho de 2016. Os dados foram analisados por meio da análise temática. Resultados: na análise dos dados elencaram-se as categorias "Concepções sobre o atendimento recebido pelos profissionais do Programa Mais Médicos na realização do pré-natal"; "A escuta qualificada, o acolhimento e o vínculo como diferencial na atenção ao pré-natal na UBS". Considerações finais: o estudo evidenciou que o atendimento realizado pelos profissionais do PMM alicerçam-se nos princípios do SUS, em que o acolhimento e o vínculo são priorizados e a escuta qualificada representa um diferencial na interação profissional usuária.

Palavras-chave: Atenção ao Pré-natal; Programa Mais Médicos; Saúde Pública.

\section{ABSTRACT}

Objective: identify the views of pregnant women on prenatal care performed by doctors of the More Doctors program in a Brazilian basic health unit (UBS). Method: this is an exploratory study with a qualitative approach. The study included 14 pregnant women attended by Brazilian public health system (SUS). Data were collected using an individual interview with a guiding questionnaire with open and closed questions, held in the nursing consultation room between June and July 2016. Data were analyzed by thematic analysis. Results: data analysis results in two categories: "Conceptions about care provided by professionals of "More Doctors" in performing prenatal"; "A qualified listening, welcoming and bonding as a differential in attention to prenatal care at UBS. " Closing remarks: the study showed that services provided by professionals are founded by SUS principles, in which the host and the bond are prioritized and qualified listening is a differential in the professional-user interaction.

Keywords: Prenatal Care; More Doctors Program; Public Health.

\section{INTRODUCÃ̃O}

A Atenção Básica é considerada a porta de entrada preferencial do Sistema Único de Saúde (SUS) que está presente em todos os municípios e próxima de todas as comunidades. É neste atendimento que $80 \%$ dos problemas de saúde são resolvidos, assim a Atenção Básica (AB) e a Estratégia Saúde da Família (ESF) constituem-se nos principais elementos que incidem na mudança paradigmática do modelo de atenção à saúde vigente. ${ }^{1}$

A consulta de pré-natal é a única forma de assegurar o desenvolvimento da gestação, permitindo o parto de um recém-nascido saudável, sem impacto para a saúde materna, minimizando os riscos para mortalidade infantil e materna, além de fatores biológicos são abordados aspectos psicossociais, preventivos e as atividades educativas. Talvez o principal indicador do prognóstico ao nascimento seja o acesso à assistência pré-natal. Os cuidados assistenciais no primeiro trimestre são utilizados como um indicador maior da qualidade 
dos cuidados. As consultas deverão ser mensais até a $28^{\mathrm{a}}$ semana, quinzenais entre a $28^{\mathrm{a}}$ e $36^{\mathrm{a}}$ semanas e semanais a partir da $36^{\text {a }}$ semana. ${ }^{2,3}$

A escassez ou ausência de profissionais médicos, interfere nesse processo, na medida em que aumenta a dificuldade em agendar uma consulta. Outro fator que interfere no atendimento à gestante é a distância de sua residência ao serviço de saúde, o que pode acarretar a ausência dessas usuárias aos serviços de saúde.

Foi com intuito de melhorar o acesso e propiciar um atendimento integral e igualitário que ocorreu a implantação do Programa Mais Médico pelo Governo Federal. Esse Programa nasceu em 2013, como uma política pública que responde ao diagnóstico identificado na Pnab, que é a consolidação e a valorização da atenção básica junto à população e aos profissionais de saúde. Faz parte de um amplo esforço do Governo Federal, com apoio de estados e municípios, visando a melhoria do atendimento aos usuários do Sistema Único de Saúde. ${ }^{1-4}$

O programa tem como objetivo resolver a questão emergencial do atendimento básico ao cidadão, mas também criar condições para continuar a garantir um atendimento qualificado no futuro para aqueles que acessam cotidianamente o SUS. Além de estender o acesso, o programa provoca melhorias na qualidade e humaniza o atendimento, com médicos que criam vínculos com seus pacientes e com a comunidade. ${ }^{4}$

A unidade básica de saúde (UBS) deve ser a porta de entrada preferencial da gestante no sistema de saúde. É o ponto de atenção estratégico para melhor acolher suas necessidades, inclusive proporcionando um acompanhamento longitudinal e continuado, principalmente durante a gravidez. ${ }^{1}$

A assistência pré-natal adequada (componente pré-natal), com a detecção e a intervenção precoce das situações de risco, bem como um sistema ágil de referência hospitalar são os grandes determinantes dos indicadores de saúde relacionados à mãe e ao bebê que têm o potencial de diminuir as principais causas de mortalidade materna e neonatal. Com a implantação do Programa no município, as consultas de pré-natal, antes realizada em um único local no município (Centro de Apoio), começaram a ser descentralizadas para as unidades básicas e estratégias da saúde da família e passaram a ser realizadas por clinico geral do programa. Este estudo alicerça-se na seguinte questão norteadora: Qual o grau de satisfação das gestantes em relação ao pré-natal, realizado por médicos do Programa Mais Médicos. O objetivo foi avaliar o grau de satisfação das gestantes, quanto ao pré-natal realizado pelos médicos do Programa Mais Médicos.

\section{MÉTODO}

Trata-se de uma pesquisa de caráter exploratório descritiva com uma abordagem qualitativa que busca avaliar o grau de satisfação das gestantes, quanto ao pré-natal realizado pelos médicos do "programa mais médicos", em uma Unidade Básica de Saúde do município de Santo Ângelo. Participaram do estudo 14 gestantes atendidas pelo SUS, que atenderam aos critérios de inclusão: ser pacientes do SUS, com diagnóstico de gravidez aceitar participar; estar em pleno domínio de suas faculdades cognitivas. Como critérios de exclusão: não ser gestante, não aceitar participar do estudo. Para a coleta de dados utilizou-se a entrevista individual com um questionário norteador, com questões abertas e fechadas, sendo realizada na sala de consulta de enfermagem da Unidade Básica de Saúde em estudo no período de julho e agosto de 2016. Os dados foram analisados por meio da análise de conteúdo temática das falas e envolveu as etapas de leitura e releitura do conteúdo dos dados narrativos para identificar temas e padrões relevantes.

Visando atender os aspectos éticos da pesquisa e tendo como base à resolução 466/2012 do Conselho Nacional de Saúde que dispõe sobre as diretrizes e normas regulamentadoras de pesquisas, envolvendo seres humanos, o projeto foi enviado ao Comitê de Ética na Pesquisa da Universidade Regional Integrada do Alto Uruguai e das Missões - Campus de Santo Ângelo. O mesmo obteve parecer favorável CAAE: 54096516.2.0000.5354. Aos sujeitos da pesquisa foi entregue o Termo de Consentimento Livre e Esclarecido que continha esclarecimentos sobre sua livre participação, preservação do anonimato e respeito à liberdade do sujeito em não participar da pesquisa. Garantia do anonimato mediante a utilização de pseudônimos, para identificação dos sujeitos. Onde foi assegurado às participantes que sua participação não teria nenhuma influência, quanto ao atendimento na rede básica de saúde.

\section{RESULTADOS E DISCUSSÃO}

Os dados emergidos mediante entrevistas com as gestantes, foram submetidos ao processo de análise, obtendo-se a seguinte categoria temática:

Concepções sobre o atendimento recebido pelos profissionais do Programa Mais Médicos na realização do pré-natal.

Nesta categoria, as gestantes apresentam suas concepções sobre o atendimento recebido na realização do pré-natal com os profissionais do Programa Mais Médicos na UBS, as quais avaliam como excelente atendimento, apresentando como diferencial a escuta atenciosa, a cortesia e paciência em esclarecer dúvidas. As afirmações, a seguir, confirmam essa percepção:

“Excelente atendimento, me senti bem segura em relação à médica, ela avalia a gestante como um todo. Vê a mãe após o parto e não só o bebê". (G7)

"Fui bem atendida, me senti acolhida e segura desde o primeiro momento". (G6)

"Os médicos estrangeiros são muito atenciosos dedicados eles têm interesse em te ajudar, tu vê isso. A doutora daqui foi muito, muito querida comigo. Quando eu fiquei sabendo que era cubana não me senti segura, mas aí, na primeira consulta mudei completamente de idéia". (G 4)

Nos discursos das gestantes evidencia-se aprovação e aceitação pelo atendimento realizados pelos profissionais do PMM, destacando-se os aspectos que 
envolvem o diálogo, a segurança e a cumplicidade que emergiu nessa relação terapêutica profissional/gestante. A escuta qualificada e o acolhimento são importantes tecnologias leves, que podem ser utilizadas como diferencial no processo de cuidado pré-natal com qualidade.

Um estudo que teve como objetivo discutir como os usuários percebem o acesso às unidades básicas de saúde, por meio do acolhimento, considera que "ao se trabalhar com a tecnologia das relações durante o atendimento, o acolhimento constitui-se como uma das primeiras ações a serem desenvolvidas por toda a equipe, no momento de receber o usuário na unidade de saúde." ${ }^{5}$

O estudo aponta que a existência de uma relação profissional-usuário satisfatória e reconhecida pelos usuários denota um grau de responsabilidade da UBS com a população, constituindo-se em uma relação de respeito, compreensão e escuta que faz a diferença entre as práticas das ações de saúde.

Nos relatos, as participantes avaliam o atendimento recebido antes da implantação do PMM como insatisfatório, centrado prioritariamente na doença, não levando em consideração outras dimensões vivenciadas pela gestante nesse período.

"Antes do programa nunca teve esse atendimento no pré-natal, as médicas cubanas têm um olhar para a mãe também, não só para o bebê, porque no convênio, se o bebê está bem, a mãe não importa". (G7)

“A atenção durante as consultas é o diferencial, porque no meu convênio não duravam mais de dez minutos. [...] Teve exames que ela pediu que meu médico do convênio não sabia do que se tratava, me senti segura". (G7)

As afirmações anteriores evidenciam que o modelo biomédico predominava nos atendimentos às gestantes na UBS, antes da chegada desses profissionais. Tal modelo não considera o ser humano em sua totalidade, na medida em que se preocupa apenas com os aspectos biológicos do atendimento, na medida em que se preocupa apenas com o exame físico da gestante. $\mathrm{Na}$ concepção das entrevistadas, o atendimento realizado pelos profissionais do PMM se processa a partir de um enfoque sistêmico, considerando a saúde como resultante de múltiplos fatores, sociais, ambientais, econômicos e subjetivos, dentre outros.

Um estudo ${ }^{5}$ que teve como objetivo conhecer o modelo de atenção à saúde, utilizado na assistência pré-natal, em unidades de Estratégia de Saúde da Família, considera relevante o processo de trabalho dos profissionais de saúde por serem "capazes de transformar a realidade de sua produção, como forma de qualificar e humanizar a assistência, bem como de fazê-la mais resolutiva, na medida em que prioriza a escuta como instrumento de trabalho, visando tornar as necessidades de cada indivíduo como centro de sua prática".

A presença da gestante, na UBS, pode ser utilizada como espaço/momento para a educação em saúde, pois "é preciso empoderar as mulheres sobre questões referentes aos seus direitos, sobre o seu corpo, condutas e procedimentos, para que se possa dar voz ativa à população e que essa busque uma assistência digna". .,6

\section{A escuta qualificada, o acolhimento e o vínculo como diferencial na atenção ao pré-natal na UBS}

As gestantes deixam transparecer em seus discursos que os profissionais do PMM, em seu processo de atendimento buscam atender de maneira qualificada, fortalecendo o vínculo e ampliando seu olhar para aspectos subjetivos que envolvem a gravidez, conforme percebido nas manifestações a seguir:

\begin{abstract}
"Ela sentou comigo, me explicou tudo sobre a evolução da gravidez, sobre o tipo de parto e não apenas os resultados dos exames. Ela se preocupa comigo". (G14)
\end{abstract}

"Eu tive aqui um atendimento melhor do que o do meu convênio". (G 3)

As gestantes avaliam como excelente o atendimento recebido no atendimento prestado pelos profissionais do PMM, reconhecendo como diferencial a disponibilidade e o interesse profissional pelo seu caso em especial. Cada usuária é respeitada em sua individualidade e autonomia, buscando-se fortalecer o vínculo e o acolhimento conforme preconizam os princípios do SUS. ${ }^{7}$ Um estudo, que buscou discutir como os usuários do município de Fortaleza (CE), percebem o acesso às unidades básicas de saúde por meio do acolhimento, que os autores observaram que "a falta de informações para os usuários sobre o funcionamento dos serviços de saúde ofertados promovem um déficit de conhecimento e causam, algumas vezes, a procura de informações nos consultórios de atendimento dos profissionais de saúde". ${ }^{5}$

\section{CONSIDERACִÕES FINAIS}

$\mathrm{O}$ estudo evidenciou que os atendimentos realizados pelos profissionais do PMM alicerçam-se nos princípios do SUS, em que o acolhimento e o vínculo são priorizados e a escuta qualificada representa um diferencial na interação profissional usuária. Acredita-se que a atitude adotada por esses profissionais no atendimento às gestantes, pode consolidar novos modos de fazer a prática na Atenção Básica, em que são priorizados aspectos que ultrapassam o modelo biomédico.

O tempo dispensado às consultas, a atenção às usuárias, segurança e o esclarecimento das dúvidas, durante o atendimento, também ficou evidente nas falas, inclusive a diferença desse atendimento aqueles que recebiam de profissionais brasileiros, mesmo os de atendimento privado.

O estudo terá continuidade na medida em que faz parte de um projeto de iniciação científica e outros achados poderão subsidiar novas análises, sobre a avaliação da atuação dos profissionais do PMM na UBS.

\section{REFERÊNCIAS}

1. Alberti GF, Schimith MD, Budó MLD, Neves GL, Rosso LF. Atributo do primeiro contato na atenção básica e práticas de cuidado: contribuições para a formação acadêmica 
do enfermeiro. Texto Contexto Enferm 2016;25(3). doi: 10.1590/0104-07072016004400014.

2. Brasil. Ministério da Saúde. Secretaria de Atenção à Saúde. Departamento de Atenção Básica. Atenção ao pré-natal de baixo risco / Ministério da Saúde. Secretaria de Atenção à Saúde. Departamento de Atenção Básica. - Brasília: Editora do Ministério da Saúde, 2012.

3. Brasil. Ministério da Saúde. Secretaria de Atenção à Saúde. Departamento de Ações Programáticas Estratégicas. Técnica de Saúde da Mulher. Pré-natal e puerpério: atenção qualificada e humanizada - manual técnico/Ministério da Saúde, Secretaria de Atenção à Saúde Departamento de Ações Programáticas Estratégicas - Brasília: Ministério da Saúde, 2005.

4. Brasil. Lei 12.871 que institui o Programa Mais Médicos. Brasília. Ministério da Saúde. 2013. Disponível em: http://
www.planalto.gov.br/ccivil_03/_Ato2011-2014/2013/Lei/ L12871.htm. > Acesso 10 de fevereiro de >.

5. Coelho MO, Jorge MSB, Araujo OME. O acesso por meio do acolhimento na atenção básica à saúde. Revista Baiana 2009:33(3):440-52.

6. Pohlmann FC, Kerber NPC, Pelzer MT, Dominguez CC, Minasi JM, Carvalho VF. Modelo de assistência pré-natal no extremo sul do país. Texto Contexto Enferm 2016;25(1). doi: 10.1590/0104-0707201600003680013.

7. Dowswell T, Carroli G, Duley L, Gates S, Gulmezoglu AM, Khan-Neelofur D, Piaggio GG. Alternative versus standard packages of antenatal care for low-risk pregnancy. Cochrane Database Syst Rev 2010;6(10). doi: 10.1002/14651858. CD000934.pub2.

Como citar: GUIMARÃES, Carine Amabile et al. Concepcões de gestantes sobre o pré-natal realizado por profissional do programa mais médicos. Cinergis, Santa Cruz do Sul, v. 18, n. 1, out. 2016. ISSN 2177-4005. Disponível em: <https://online.unisc.br/seer/index.php/cinergis/article/view/8144>. Acesso em: 11 out. 2016. doi:http://dx.doi.org/10.17058/cinergis. v18i1.8144. 